\title{
Caractéristiques phénotypique et structurale de la population de Parkia biglobosa (Jacq.) R. Br. du Nord-Bénin
}

\author{
Kourouma KOURA*, Yéba MBAIDE et Jean Cossi GANGLO \\ Laboratoire des Sciences Forestières, Faculté des Sciences Agronomiques, Université d'Abomey-Calavi, \\ 01 BP 526 Cotonou, Bénin. \\ *Auteur correspondant ; E-mail : kourakourouma@yahoo.fr ; Tél : +22996716130
}

\section{RESUME}

La présente étude a pour objectif principal d'identifier les caractéristiques phénotypiques et structurales des sous-populations de Parkia biglobosa (néré) du Nord-Bénin, en vue d'une gestion durable de l'espèce. Cinq cent quatre vingt seize arbres de néré ont été échantillonnés. Les principaux résultats ont montré qu'il existe quatre sous-populations de néré dans le milieu d'étude. Les huit descripteurs quantitatifs étudiés sur l'espèce (nombre de rachis par feuille, nombre de gousses par infrutescence, longueur du pédoncule, épaisseur du pédoncule, longueur du pédicelle de la gousse, longueur de la gousse, poids de la gousse, nombre de graines par gousse) varient d'une sous-population à une autre, alors que les paramètres dendrométriques varient très peu. L'étude de la structure diamétrique des différentes sous-populations a montré que l'espèce connait un problème de régénération. En ce qui concerne l'importance pratique de ce travail, notons que l'aspect du néré le plus recherché est sa production fruitière. Les pieds de néré de la sous-population Kalalé présentent des valeurs moyennes élevées de poids de gousse, de nombre de graines par gousse et de nombre de gousses par infrutescence. Ils sont particulièrement intéressants et peuvent être pris en compte dans le cadre d'une sélection de variétés à haut rendement.

(C) 2013 International Formulae Group. All rights reserved.

Mots clés : Sous-population, Parkia biglobosa, phénotype, structure, Nord-Bénin.

\section{INTRODUCTION}

Au Bénin, Parkia biglobosa fait partie des plantes génératrices de Produits Forestiers Non Ligneux les plus importantes (Eyog-Matig et al., 1999, 2002). Il rend d'énormes services à une grande partie de la population rurale, le plus souvent, comme principale source de revenus et moyen efficace de lutte contre la pauvreté (Gutierrez, 2000). En effet, Parkia biglobosa est l'une des essences d'intérêt socio-économique les plus valorisées par les populations locales du Nord-Bénin (Baco, 2007). Parmi les trente une (31) espèces ligneuses médicinales les plus utilisées dans la médecine traditionnelle béninoise, elle occupe la cinquième position (Eyog-Matig et al., 2002). En outre, de nombreux travaux ont confirmé sa grande valeur nutritive et la place importante qu'elle occupe dans l'alimentation au Bénin (EyogMatig et al., 2002 ; Gutierrez, 2000 ; Djakpo, 2005). La pulpe de ses gousses est riche en saccharose et ses graines sont riches en glucides, protides et lipides constituant ainsi un excellent aliment énergétique (Buisson, 1965 ; Djakpo, 2005). C'est pourquoi, dans la 
liste des dix huit (18) espèces ligneuses alimentaires prioritaires à conserver (FAO, 2001), elle occupe le quatrième rang. Parkia biglobosa est aussi une espèce agroforestière importante (Bonkougou, 1987; Lafleur, 2008). Associé aux cultures agricoles, elle contribue à l'enrichissement du sol et à l'augmentation des rendements. A propos de cette espèce, tout, de la racine à la cime, contribue à la survie de l'homme (Rateau, 1995).

Cependant, malgré le rôle important que jouent les ressources forestières en général et Parkia biglobosa en particulier dans l'amélioration des conditions de vie humaine au Bénin, leur maintien à long terme suscite quelques inquiétudes, compte tenu de la dégradation rapide et actuelle des surfaces forestières dans le pays (Schmidt-Soltau et Alimi, 2008 ; FAO, 2010). Les menaces qui pèsent sur les espèces ligneuses au Bénin sont réelles et préoccupantes. Ces menaces sont liées essentiellement aux prélèvements intenses, aux mutilations, à la déforestation incontrôlée ou mal gérée et aux pratiques agricoles dévastatrices. Actuellement, le Bénin se situe parmi les pays du monde où les superficies qui subissent de déforestation sont élevées, avec une perte annuelle de 50000 ha entre 2000 et 2010 (FAO, 2010). C'est ainsi que Parkia biglobosa, faisant partie de ce vaste ensemble est particulièrement touché. En effet, on assiste au Bénin, depuis un certain temps, au vieillissement de ses populations, alors que la régénération naturelle de l'espèce, très faible, ne permet pas d'assurer leur renouvellement (Bonkoungou, 1987 ; Gbédji, 2003). Les études ont montré une diminution de son aire de répartition au Bénin (Gutierrez et JuhéBeaulaton, 2002). Compte tenu de l'importance socio-économique et culturelle de Parkia biglobosa, les populations béninoises ont tendance à récolter l'ensemble de la production fruitière limitant ainsi la régénération de l'espèce (Eyog-Matig et al., 2002 ; Sina, 2006).
Les études réalisées jusque-là sur Parkia biglobosa au Bénin concerne surtout l'aspect de la technologie alimentaire (Hounhouigan et al., 2004 ; Djakpo, 2005 ; Azokpota et al., 2006), l'aspect commercial (Gutierrez, 2000), l'aspect ethnobotanique (Koura, 2003), l'histoire, la morphologie et la structure de ses différents parcs (Agbahungba et Depommier, 1989; Gutierrez et JuhéBeaulaton, 2002 ; Gbédji, 2003). Malgré ces travaux, plusieurs questions restent sans réponse notamment dans le Nord-Bénin, zone de prédilection de Parkia biglobosa au Bénin : (1) Quelles sont les différentes souspopulations de Parkia biglobosa du NordBénin ? (2) Quelles sont les caractéristiques dendrométriques et structurales de chaque sous-population identifiée ? Le présent travail vise principalement à caractériser la population de Parkia biglobosa du NordBénin en vue de répondre à ces questions. Ainsi, les objectifs spécifiques de l'étude consistent à : (i) identifier les différentes souspopulations naturelles de Parkia biglobosa du Nord-Bénin en se basant sur des descripteurs phénotypiques quantitatifs ; (ii) déterminer les caractéristiques dendrométriques et structurales de chaque sous-population identifiée.

\section{MATERIEL ET METHODES Milieu d'étude}

La présente étude a été réalisée dans les parcs à Parkia biglobosa les plus représentatifs du Nord-Bénin. Le Nord-Bénin comporte quatre départements (subdivisions administratives): l'Alibori, le Borgou, l'Atacora et la Donga. L'Alibori et le Borgou occupent la partie Nord-Est du Bénin tandis que l'Atacora et la Donga sont situés au NordOuest du Bénin. Ces quatre départements occupent une superficie totale de $83723 \mathrm{~km}^{2}$ soit $74 \%$ de la superficie totale du Bénin $\left(112622 \mathrm{~km}^{2}\right)$. Ils sont divisés en vingt sept (27) communes. Les précipitations sont unimodales dans l'ensemble des communes. Les sols sont soit hydromorphes, bien drainés, soit ferrugineux et de fertilité variable. La 
température moyenne annuelle est de $35{ }^{\circ} \mathrm{C}$. La moyenne annuelle des précipitations est inférieure à $1100 \mathrm{~mm}$. La végétation est soit composée de savanes avec de petits arbres, soit caractérisée par une mosaïque de forêts claires, de forêts denses et de savanes arbustives à arborées avec des galeries forestières. Les activités génératrices de revenus menées dans ces communes comprennent l'agriculture, l'élevage, la pêche, la chasse, la transformation alimentaire, le commerce et l'artisanat. La transformation alimentaire est principalement pratiquée par les femmes individuellement ou en groupes avec un équipement artisanal. Les principaux produits transformés sont les noix de Vitellaria paradoxa C. F. Gaertn. (transformées en beurre), les graines de Parkia biglobosa (transformées en condiment alimentaire), les graines de Sorghum bicolor (L.) Moench (transformées en boisson alcoolisée), les gousses d'Arachis hypogea L. (transformées en huile).

Méthode utilisée pour identifier les souspopulations de Parkia biglobosa du NordBénin

Les parcs à Parkia biglobosa retenus dans la présente étude répondent aux critères suivants : peuplements non dégradés ou non menacés de disparition; effectif assez élevé en pieds de Parkia biglobosa; possibilité d'observation sur plusieurs années; station accessible en toutes saisons (Frankie et al., 1974 ; Ouédraogo, 1995). Six (06) communes dont les peuplements sont les plus représentatifs ont été retenues. Il s'agit des communes de Matéri, Toucountouna et Natitingou dans le département de l'Atacora, Parakou, Sinendé et Kalalé dans le département du Borgou. Cinquante neuf (59) placeaux de formes et de superficies variables (entre 0,1099 ha et 4,5 ha) ont été installés (Figure 1) de manière à ce que chaque placeau contienne au moins 10 arbres de Parkia biglobosa de diamètre à hauteur de poitrine $(1,30 \mathrm{~m} \mathrm{du}$ sol) supérieur ou égal à $10 \mathrm{~cm}$
(Duplat et Perrote, 1981). Cinq cent quatre vingt seize (596) arbres de Parkia biglobosa ont été au total échantillonnés. Les coordonnées GPS de chaque arbre de Parkia biglobosa échantillonné ont été relevées.

Les analyses pour la détermination des sous-populations de Parkia biglobosa du Nord-Bénin ont été réalisées sur les valeurs moyennes par placeau de différents descripteurs quantitatifs mesurés sur les pieds de Parkia biglobosa ayant un diamètre à 1,30 $\mathrm{m}$ du sol (dbh) supérieur ou égal à $10 \mathrm{~cm}$. Ces descripteurs sont :

- les infrutescences. En suivant les axes de référence Est-Ouest, Nord-Sud du houppier de chaque arbre, une infrutescence a été choisie à l'extrémité Est du diamètre du houppier, une à l'extrémité Ouest, une à l'extrémité Nord, une à l'extrémité Sud et enfin une au centre. De plus une infrutescence a été choisie vers le sommet de l'arbre, une vers la base de la cime et une vers la partie médiane de la cime. Sur chaque infrutescence prélevée, le nombre de gousses a été compté ; puis la longueur et l'épaisseur du pédoncule ont été mesurées.

- les gousses (fruits). Pour la caractérisation des gousses, cinquante pour cent $(50 \%)$ des gousses de chaque infrutescence portant des numéros impairs au comptage, ont été utilisés. Ainsi sur les gousses portant des nombres impairs au comptage, la longueur du pédicelle a été mesurée, de même que la longueur de la gousse et le poids de la gousse.

Lors de la caractérisation des gousses, le nombre de graines par gousse a été également compté.

- les feuilles. Les branches portant les infrutescences (choisies ci-dessus) puis celles situées immédiatement à droite, et ne portant pas d'infrutescences, ont été coupées et descendues. Sur chaque branche descendue, les feuilles ont été comptées et sur chaque feuille portant des nombres impairs au comptage, le nombre de paires de rachis a été compté. 
Pour chaque arbre, une fiche a été remplie et comporte la synthèse des informations recueillies sur les descripteurs. Les modalités des différents paramètres ont été codifiées. L'analyse de l'organisation de la variabilité a été réalisée à l'aide d'une Analyse Factorielle Discriminante effectuée avec le logiciel SAS 9.2. L'analyse de variance multiple (MANOVA) a été utilisée pour tester si les variations observées sur l'ensemble des caractères quantitatives mesurées sont significatives (Hill et Lewicki, 2006). Ceci a été complété par une analyse de variance simple (ANOVA) et le test de comparaison de moyennes de Newman-Keuls. Les liens entre les diverses variables ont été déterminés en utilisant les coefficients de corrélation.

\section{Méthode de caractérisation dendrométrique des sous-populations}

Les caractéristiques dendrométriques des sous-populations ont été prises sur tous les individus de diamètre à $1,30 \mathrm{~m}$ du sol, supérieur à $10 \mathrm{~cm}$ (soit $31,4 \mathrm{~cm}$ de circonférence à $1,30 \mathrm{~m}$ du sol). Les données ont été collectées dans les placeaux utilisés pour l'identification et la caractérisation des sous-populations et se rapportent aux paramètres suivants : la circonférence à hauteur de poitrine (1,30 $\mathrm{m} \mathrm{du} \mathrm{sol})$, la hauteur totale et la hauteur fût. Lors de la détermination de la hauteur totale, deux visées ont été effectuées à l'aide d'un clinomètre : une première visée $\left(\mathrm{V}_{1}\right)$, au sommet de l'arbre, évaluée en pourcentage de la distance (L) de l'opérateur à l'arbre ; une deuxième visée $\left(\mathrm{V}_{2}\right)$ au pied de l'arbre et également évaluée en pourcentage de la distance (L) de l'opérateur à l'arbre. La hauteur totale (h) de l'arbre mesurée est obtenue par la relation $\mathrm{h}$ $=\left[\left(V_{1}-V_{2}\right) * L\right] / 100$. En ce qui concerne la hauteur fût, la même démarche a été suivie, seulement que la première visée $\left(\mathrm{V}_{1}\right)$ a été réalisée au niveau où s'insère la première grosse branche.
En dehors des paramètres dendrométriques mentionnés ci-dessus et directement mesurés sur les arbres, les paramètres dendrométriques suivants ont été calculés :

- la densité du peuplement (N, en arbres/ha) : c'est le nombre de pieds de Parkia biglobosa recensés à l'hectare. Elle a été calculée en utilisant la formule : $\mathrm{N}=\frac{n}{s}$ avec $n$, le nombre de pieds recensés sur une superficie $s$ donnée.

- la surface terrière : elle est déterminée par la formule :

$\mathrm{G}=\sum_{i=1}^{n} \pi d_{i}^{2} / 4$ ou $\mathrm{G}=\sum_{i=1}^{n} c_{i}^{2} / 4 \pi$

avec $\mathrm{G}$, la surface terrière des arbres du placeau en $\mathrm{m}^{2} / \mathrm{ha} ; \mathrm{d}_{\mathrm{i}}$, le diamètre $(\mathrm{m})$ à $1,30 \mathrm{~m}$ du sol de l'arbre i du placeau considéré et $\mathrm{c}_{\mathrm{i}}$, la circonférence $(\mathrm{m})$ à 1,30 m du sol de l'arbre i du placeau considéré $(\mathrm{m})$.

- le diamètre Dg de l'arbre de surface terrière moyenne: il a été obtenu par la formule :

$\mathrm{Dg}=\sqrt{\frac{\sum d i^{2}}{n}}$ avec $d i$ le diamètre en $\mathrm{cm}$ de l'arbre i du placeau considéré et $n$ le nombre d'arbres dans le placeau.

La comparaison des sous-populations suivant les différents paramètres dendrométriques mesurés ou calculés a été faite grâce à l'Analyse de variance suivie du test de comparaison de moyennes de Newman-Keuls.

\section{Méthode de caractérisation structurale des sous-populations identifiés}

Les pieds de Parkia biglobosa appartenant à chaque sous-population ont été répartis par classes de diamètre d'amplitude $10 \mathrm{~cm}$. Des histogrammes traduisant la répartition par classe de diamètre des pieds de Parkia biglobosa de chaque sous-population ont été tracés à l'aide du tableur Excel. 


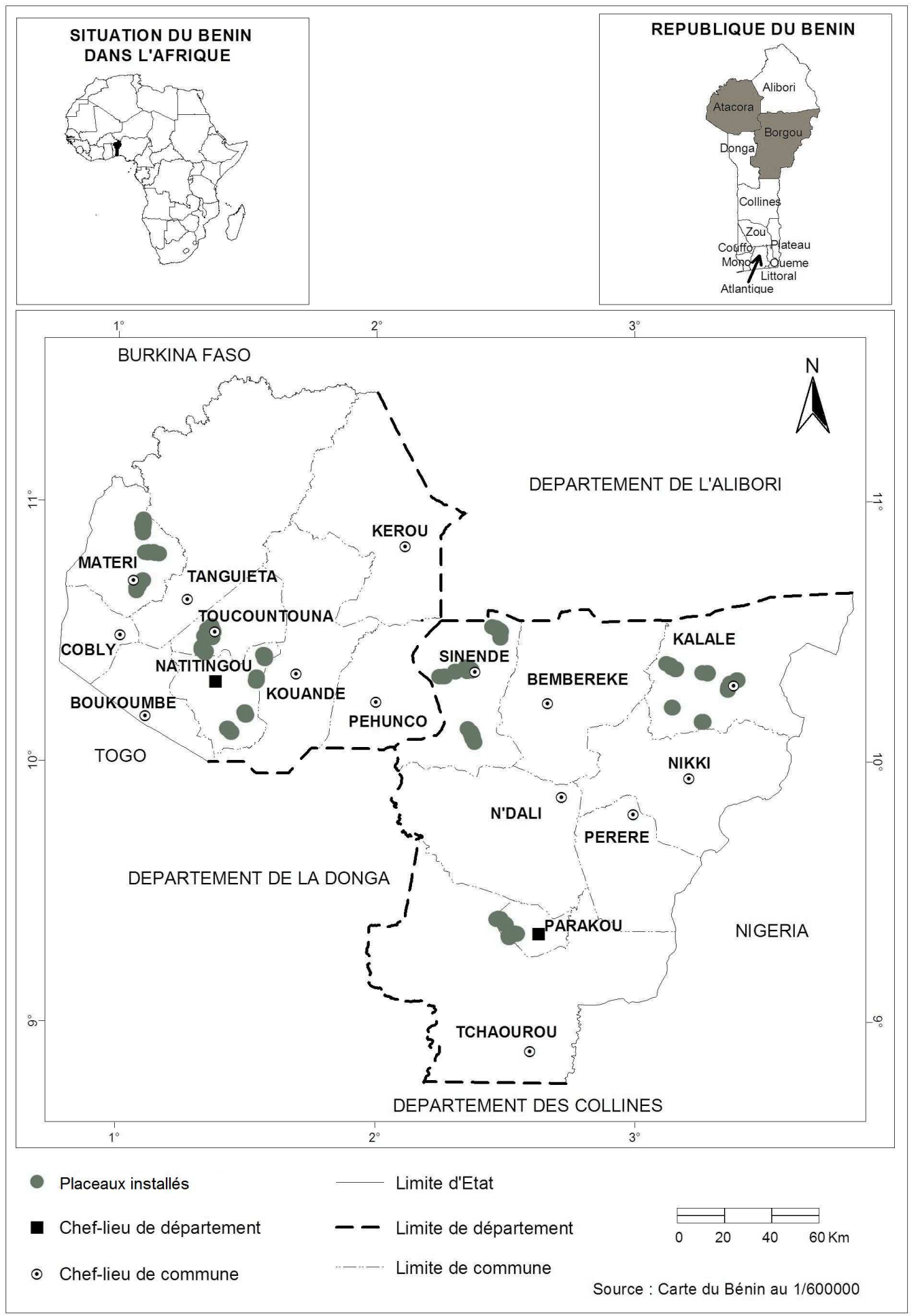

Figure 1 : Localisation géographique des placeaux installés dans les peuplements naturels de Parkia biglobosa de la zone d'étude. 


\section{RESULTATS}

Identification des sous-populations de néré Relations entre les différents descripteurs

L'examen de la matrice de corrélation sur la totalité de l'échantillon (Tableau 1) indique l'existence de quelques corrélations fortes, positives et très significatives entre les différents descripteurs quantitatifs mesurés sur Parkia biglobosa. Nous observons une forte corrélation positive entre la longueur de la gousse et le poids de la gousse $(r=0,88)$, entre le poids de la gousse et le nombre de graines $(r=0,85)$, entre la longueur de la gousse et le nombre de graines $(r=0,78)$, entre le poids de la gousse et l'épaisseur du pédoncule $(r=0,69)$, entre le nombre de graines et l'épaisseur du pédoncule $(r=0,69)$, entre la longueur de la gousse et l'épaisseur du pédoncule $(r=0,65)$, entre le nombre de rachis et l'épaisseur du pédoncule $(r=0,52)$. Chez Parkia biglobosa, le poids de la gousse augmente avec la longueur de la gousse, le nombre de graines par gousse et l'épaisseur du pédoncule.

\section{Les sous-populations identifiées}

Les valeurs moyennes par placeau des descripteurs quantitatifs mesurés ont été soumises à une Analyse Factorielle Discriminante. Le Tableau 2 récapitule les valeurs propres des différents axes canoniques au terme de l'Analyse Factorielle Discriminante. Les trois axes canoniques expliquent $100 \%$ de la variance totale tandis que les deux premiers axes canoniques en expliquent $96,44 \%$. Ces deux premiers axes serviront donc à décrire les sous-populations. La projection des différentes moyennes par placeau sur le système d'axes canoniques 1 et 2 (Figure 2) a permis de distinguer quatre (4) groupes différents correspondant à quatre (4) sous-populations de Parkia biglobosa $\left(\mathrm{R}^{2}=\right.$ 0,51):

- Sous-population 1 (E1): elle est majoritairement constituée des arbres de la commune de Toucountouna et constitue de ce fait la «Sous-population Toucountouna ».

- Sous-population 2 (E2) : elle regroupe majoritairement les arbres de la commune de Kalalé. Dans la suite du travail, elle est dénommée «Sous-population Kalalé ».

- Sous-population 3 (E3) : elle est composée majoritairement des arbres de la commune de Sinendé et constitue dans la suite du travail la « Sous-population Sinendé ».

- Sous-population 4 (E4) : elle est composée majoritairement des arbres de la commune de Matéri. Dans la suite du travail, elle représente la «Sous-population Matéri ».

L'analyse de la corrélation entre les descripteurs quantitatifs et les deux axes canoniques de l'Analyse Discriminante Décisionnelle (Tableau 3) montre que l'axe 1 est positivement corrélé à la longueur des gousses (lgo) ( $\mathrm{r} \approx 0,83$ ), au poids des gousses (pgo) $\quad(\mathrm{r}=0,89)$, au nombre de graines par gousse (ngr) $(\mathrm{r} \approx 0,93)$, à l'épaisseur du pédoncule des infrutescences (epd) $(\mathrm{r}=0,99)$, au nombre de rachis par feuille (nra) ( $\mathrm{r}=$ $0,79)$, au nombre de gousses par infrutescence (ngo) ( $\mathrm{r} \approx 0,50)$, tandis que l'axe canonique 2 est positivement corrélé à la longueur du pédicelle $(\mathrm{lpl})(\mathrm{r}=0,93)$, à la longueur du pédoncule des infrutescences (lpd) $(\mathrm{r}=0,95)$ et à la longueur des gousses $(\operatorname{lgo})(\mathrm{r} \approx 0,56)$. Il faut noter que le descripteur «nombre de rachis par feuille » est négativement corrélé avec le deuxième axe canonique $(r=-0,61)$. La plupart des descripteurs mesurés (lgo, pgo, epd, ngr, lpl, lpd et nra) ont des valeurs élevées pour la sous-population Kalalé alors que leurs valeurs sont faibles pour la souspopulation Toucountouna.

Les distances de Mahalanobis entre les quatre (4) sous-populations obtenues sont toutes significatives, ce qui confirme que les sous-populations se distinguent bien les unes des autres (Tableau 4). 
Caractérisation des sous-populations

Caractéristiques phénotypiques des souspopulations de néré du Nord-Bénin

Le Tableau 5 présente les valeurs moyennes et les erreurs standards des différents descripteurs par sous-population. L'Analyse de la variance multiple (MANOVA) réalisée sur l'ensemble des descripteurs étudiés a donné un résultat hautement significatif (Lambda de Wilks = $0,025 ; \mathrm{p}<0,0001)$. Il est à noter que les arbres de néré de la sous-population Toucountouna présentent des valeurs faibles pour la plupart des descripteurs.

Le poids de la gousse varie de façon hautement significative d'une sous-population à une autre ( $\mathrm{p}<0,0001)$. La sous-population Kalalé présente les valeurs moyennes de poids de gousse les plus élevées $(13,92 \pm 0,44 \mathrm{~g})$ tandis que les valeurs moyennes les plus faibles se rencontrent au niveau de la souspopulation Toucountouna $(8,80 \pm 0,58 \mathrm{~g})$.

Le nombre de graines par gousse varie de façon hautement significative d'une souspopulation à une autre $(\mathrm{p}<0,0001)$. Les souspopulations Kalalé et Sinendé ont les valeurs moyennes de nombre de graines par gousse les plus élevées (respectivement 16,73 \pm 0,22 et $16,08 \pm 0,31$ ) tandis que la valeur la plus faible se situe au niveau de la sous-population Toucountouna $(12,59 \pm 0,45)$.

Le nombre de gousses par infrutescence varie de façon hautement significative d'une sous-population à une autre $(p<0,0001)$. Les valeurs moyennes de nombre de gousses par infrutescence les plus élevées se trouvent au niveau des souspopulations Kalalé, Sinendé et Matéri (respectivement 4,97 $\pm 0,33 ; 5,44 \pm 0,29$ et $5,64 \pm 0,43)$ tandis que la valeur la plus faible se retrouve au niveau de la sous-population Toucountouna $(3,55 \pm 0,30)$.

Il apparait ici que les pieds de Parkia biglobosa de la sous-population Kalalé présentent des valeurs moyennes élevées de poids de gousse, de nombre de graines par gousse et de nombre de gousses par infrutescence. Ils sont donc particulièrement intéressants et peuvent être pris en compte dans le cadre d'une sélection de variétés à haut rendement.

Caractéristiques structurales des souspopulations de néré du Nord-Bénin

Les structures diamétriques des populations de Parkia biglobosa des différentes sous-populations identifiées (Figures $3 ; 4 ; 5$ et 6 ) montrent une faible densité des pieds de néré dans les classes inférieures de diamètre. Le Tableau 6 présente la synthèse des paramètres dendrométriques déterminés pour les différentes souspopulations identifiées. La densité moyenne des arbres de néré de ces sous-populations varie de 13 pieds/ha à 22 pieds/ha. La surface terrière moyenne quant à elle, varie de 2,47 $\mathrm{m}^{2} /$ ha à $5,10 \mathrm{~m}^{2} /$ ha. Le diamètre de l'arbre de surface terrière moyenne varie de $46,79 \mathrm{~cm}$ à $56,70 \mathrm{~cm}$. La hauteur totale moyenne varie de $11,31 \mathrm{~m}$ à 14,55 m tandis que la hauteur fût moyenne varie de $1,89 \mathrm{~m}$ à $2,44 \mathrm{~m}$. Le Tableau 6 rend également compte des résultats de l'analyse de variance simple effectuée pour comparer les différentes souspopulations identifiées. Cette analyse de variance a permis de faire ressortir les ressemblances et dissemblances qui existent entre ces différentes sous-populations. Toutes les sous-populations sont analogues du point de vue de leur densité et de leur surface terrière ( $\mathrm{p}$ respectivement égal à 0,379 et $0,572$ donc supérieur à 0,05$)$. Nous concluons que ces paramètres ne sont pas discriminatoires pour ces sous-populations. En ce qui concerne le diamètre de l'arbre de surface terrière moyenne (Dg), la hauteur totale et la hauteur fût, les sous-populations sont significativement différentes les unes des autres au seuil de $5 \%$ (p respectivement égal à $0,020 ; 0,000$ et 0,023 donc $\mathrm{p}<0,05)$. 
Tableau 1 : Matrice de corrélation sur la totalité de l'échantillon.

\begin{tabular}{|c|c|c|c|c|c|c|c|c|}
\hline Descripteurs & Ipl & lgo & pgo & ngr & ngo & lpd & epd & nra \\
\hline lpl & 1 & & & & & & & \\
\hline $\operatorname{lgo}$ & 0,23 & 1 & & & & & & \\
\hline pgo & 0,23 & $0,88 * *$ & 1 & & & & & \\
\hline ngr & 0,24 & $0,78 * *$ & $0,85^{* *}$ & 1 & & & & \\
\hline ngo & $0,38 * *$ & $0,27 *$ & $0,35^{* *}$ & $0,39 * *$ & 1 & & & \\
\hline lpd & $0,30^{*}$ & 0,07 & 0,16 & 0,16 & 0,10 & 1 & & \\
\hline epd & $0,27 *$ & $0,65^{* *}$ & $0,69 * *$ & $0,69 * *$ & $0,39 * *$ & 0,13 & 1 & \\
\hline nra & $-0,19$ & 0,10 & $0,23^{*}$ & $0,29 *$ & 0,03 & $-0,05$ & $0,52 * *$ & 1 \\
\hline
\end{tabular}

Tableau 2 : Valeurs propres et variances expliquées des deux axes canoniques de l'Analyse Discriminante Décisionnelle.

\begin{tabular}{lccc}
\hline Axes canoniques & $\begin{array}{c}\text { Valeurs } \\
\text { propres }\end{array}$ & Variances expliquées & Variances cumulées \\
\hline 1 & 7,5156 & $73,15 \%$ & $73,15 \%[\mathrm{p}<0,0001]$ \\
2 & 2,3930 & $23,29 \%$ & $96,44 \%[\mathrm{p}<0,0001]$ \\
3 & 0,3658 & $3,56 \%$ & $100 \%[\mathrm{p}=0,0128]$ \\
\hline \multicolumn{3}{c}{$\mathrm{p}=$ niveau de signification }
\end{tabular}

Tableau 3 : Corrélations entre les descripteurs quantitatifs et les deux axes canoniques.

\begin{tabular}{ccc}
\hline Descripteurs & $\begin{array}{c}\text { Axe canonique 1 }(\mathbf{0 , 7 3} ; \\
\mathbf{p}<\mathbf{0 , 0 0 0 1})\end{array}$ & $\begin{array}{c}\text { Axe canonique 2 }(\mathbf{0 , 2 3} ; \\
\mathbf{p}<\mathbf{0 , 0 0 0 1})\end{array}$ \\
\hline lpl & 0,3455 & 0,9318 \\
lgo & 0,8282 & 0,5569 \\
pgo & 0,8933 & 0,4495 \\
ngr & 0,9249 & 0,3582 \\
ngo & 0,4994 & 0,4487 \\
lpd & 0,0009 & 0,9543 \\
epd & 0,9933 & 0,1154 \\
nra & 0,7908 & $-0,6120$ \\
\hline
\end{tabular}

Tableau 4 : Distances de Mahalanobis entre les sous-populations.

\begin{tabular}{lcccc}
\hline Ecotypes & Toucountouna & Kalalé & Sinendé & Matéri \\
\hline Toucountouna & 0 & & & \\
Kalalé & $41,04(\mathrm{p}<0,0001)$ & 0 & 0 & \\
Sinendé & $33,75(\mathrm{p}<0,0001)$ & $10,66(\mathrm{p}<0,0001)$ & & \\
Matéri & $11,03(\mathrm{p}<0,0001)$ & $24,82(\mathrm{p}<0,0001)$ & $32,74(\mathrm{p}<0,0001)$ & 0 \\
\hline \multicolumn{4}{c}{$\mathrm{p}=$ niveau de signification }
\end{tabular}


Tableau 5 : Valeurs moyennes et erreurs standards des différents descripteurs par sous-population.

\begin{tabular}{|c|c|c|c|c|c|c|c|c|}
\hline Sous populations & $\begin{array}{c}\text { lpl }(\mathrm{cm}) \\
(\mathrm{p}<\mathbf{0 , 0 0 0 1 )}\end{array}$ & $\begin{array}{c}\operatorname{lgo}(\mathrm{cm}) \\
(\mathrm{p}<0,0001)\end{array}$ & $\begin{array}{c}\text { pgo }(g) \\
(p<0,0001)\end{array}$ & $\begin{array}{c}\text { ngr } \\
(\mathrm{p}<0,0001)\end{array}$ & $\begin{array}{c}\text { ngo } \\
(\mathbf{p}<0,0001) \\
\end{array}$ & $\begin{array}{c}\text { lpd }(\mathrm{cm}) \\
(\mathrm{p}<0,0001)\end{array}$ & $\begin{array}{c}\text { epd }(\mathrm{cm}) \\
(\mathrm{p}<0,0001)\end{array}$ & $\begin{array}{c}\text { nra } \\
(\mathbf{p}<0,0001)\end{array}$ \\
\hline $\begin{array}{l}\text { Sous-population } \\
\text { Toucountouna }\end{array}$ & $3,30 \pm 0,13 \mathrm{a}$ & $17,88 \pm 0,64 \mathrm{a}$ & $8,80 \pm 0,58 \mathrm{a}$ & $12,59 \pm 0,45 a$ & $3,55 \pm 0,30 \mathrm{a}$ & $17,27 \pm 0,51 \mathrm{a}$ & $0,48 \pm 0,02 \mathrm{a}$ & $16,25 \pm 0,25 b$ \\
\hline $\begin{array}{l}\text { Sous-population } \\
\text { Kalalé }\end{array}$ & $3,99 \pm 0,15 \mathrm{c}$ & $23,71 \pm 0,37 \mathrm{c}$ & $13,92 \pm 0,44 \mathrm{~d}$ & $16,73 \pm 0,22 \mathrm{c}$ & $4,97 \pm 0,33 \mathrm{~b}$ & $19,51 \pm 0,33 b$ & $1,39 \pm 0,03 \mathrm{c}$ & $16,51 \pm 0,16 b$ \\
\hline $\begin{array}{l}\text { Sous-population } \\
\text { Sinendé }\end{array}$ & $3,57 \pm 0,07 \mathrm{ab}$ & $21,80 \pm 0,25 b$ & $12,41 \pm 0,29 \mathrm{c}$ & $16,08 \pm 0,31 \mathrm{c}$ & $5,44 \pm 0,29 b$ & $16,88 \pm 0,35 \mathrm{a}$ & $1,33 \pm 0,06 \mathrm{c}$ & $17,51 \pm 0,11 \mathrm{c}$ \\
\hline $\begin{array}{l}\text { Sous-population } \\
\text { Matéri }\end{array}$ & $3,87 \pm 0,12 b c$ & $20,86 \pm 0,44 \mathrm{~b}$ & $10,93 \pm 0,24 b$ & $14,53 \pm 0,27 \mathrm{~b}$ & $5,64 \pm 0,43 \mathrm{~b}$ & $18,61 \pm 0,49 b$ & $0,68 \pm 0,04 \mathrm{~b}$ & $15,46 \pm 0,20 \mathrm{a}$ \\
\hline
\end{tabular}

Les valeurs qui sont suivies de la même lettre dans une même colonne ne sont pas significativement différentes au seuil de probabilité de 5\%.

Tableau 6 : Caractéristiques dendrométriques des différentes sous-populations identifiées.

\begin{tabular}{lccccc}
\hline Sous-populations & Densité $(\mathbf{N} / \mathbf{h a})$ & $\mathbf{G}\left(\mathbf{m}^{2} / \mathbf{h a}\right)$ & Dg $(\mathbf{c m})$ & Htot $(\mathbf{m})$ & Hfût $(\mathbf{m})$ \\
\hline Sous-population & & & & & \\
Toucountouna & $22,30(16,03) \mathrm{a}$ & $4,18(2,82) \mathrm{a}$ & $49,50(8,23) \mathrm{ab}$ & $11,86(1,50) \mathrm{a}$ & $1,95(0,48) \mathrm{ab}$ \\
Sous-population Kalalé & $13,30(7,03) \mathrm{a}$ & $3,40(1,97) \mathrm{a}$ & $55,95(11,45) \mathrm{b}$ & $14,55(1,61) \mathrm{b}$ & $2,04(0,43) \mathrm{ab}$ \\
Sous-population Sinendé & $17,38(20,03) \mathrm{a}$ & $5,10(8,85) \mathrm{a}$ & $56,70(11,01) \mathrm{b}$ & $14,35(1,71) \mathrm{b}$ & $2,44(0,65) \mathrm{b}$ \\
Sous-population Matéri & $13,43(11,64) \mathrm{a}$ & $2,47(2,55) \mathrm{a}$ & $46,79(8,65) \mathrm{a}$ & $11,31(1,62) \mathrm{a}$ & $1,89(0,59) \mathrm{a}$ \\
\hline
\end{tabular}




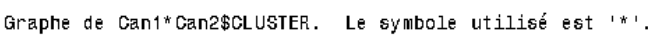

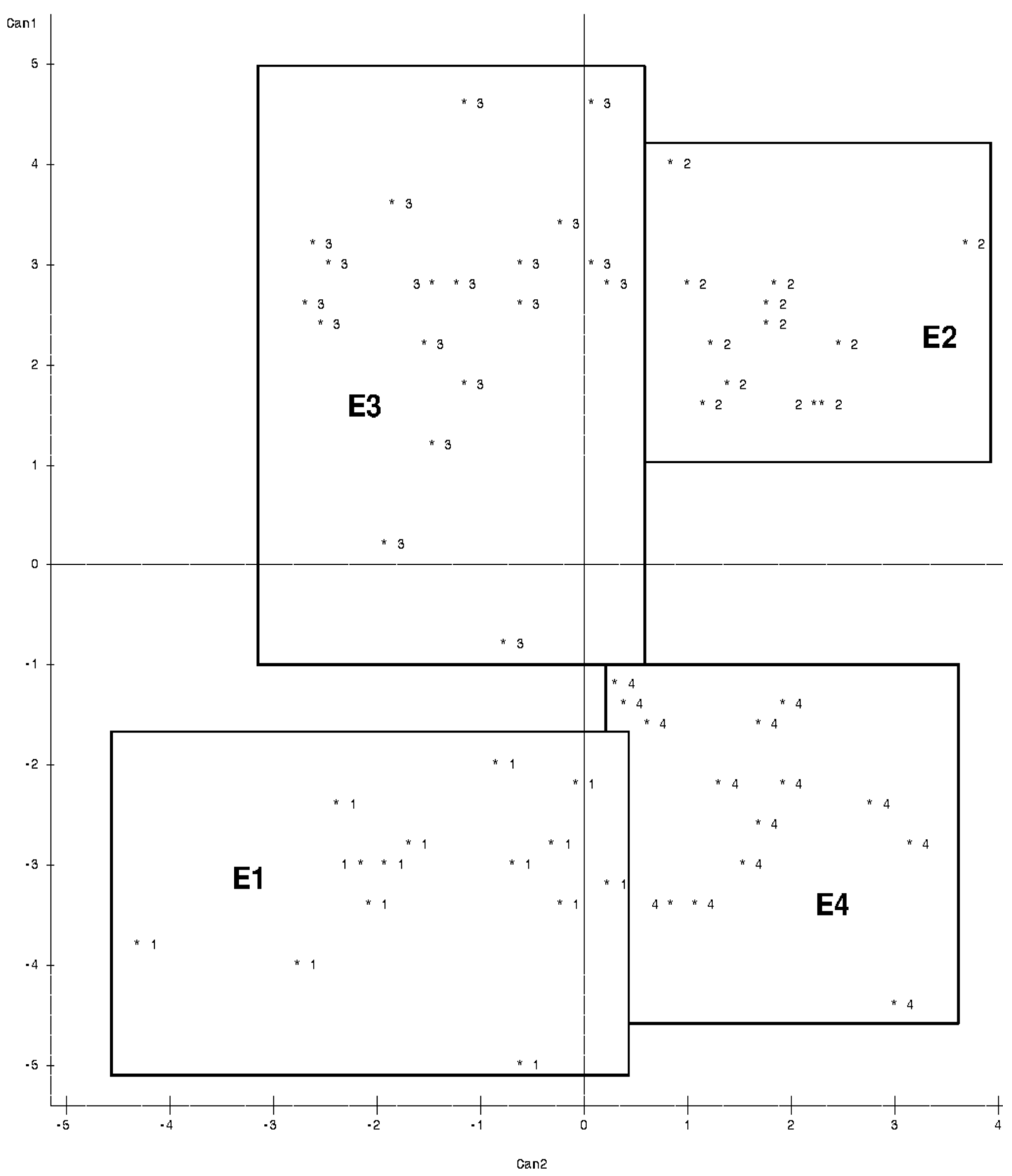

Figure 2 : Représentation des sous-populations identifiées dans le système d'axes canoniques 1 et 2. 


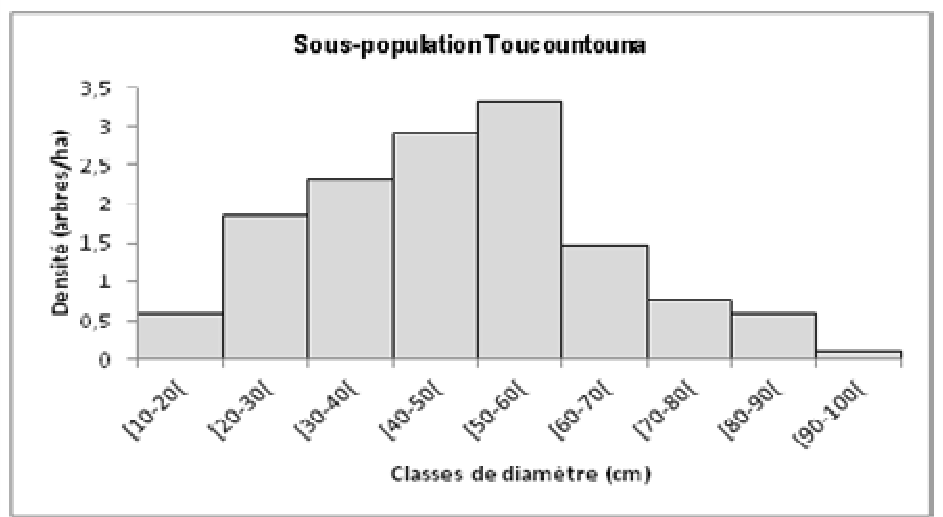

Figure 3 : Structure diamétrique des pieds de P. biglobosa de la sous-population Toucountouna.

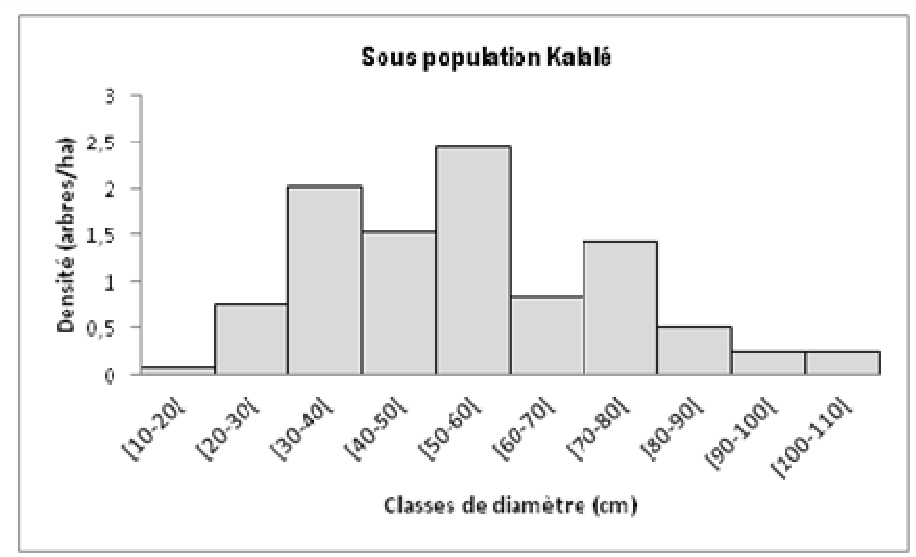

Figure 4 : Structure diamétrique des pieds de $P$. biglobosa de la sous-population Kalalé.

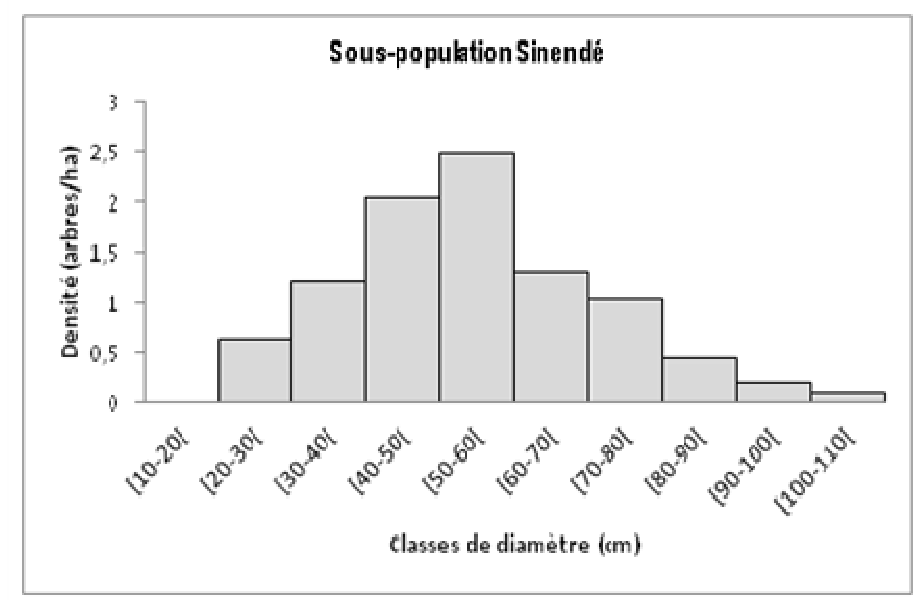

Figure 5 : Structure diamétrique des pieds de $P$. biglobosa de la sous-population Sinendé. 


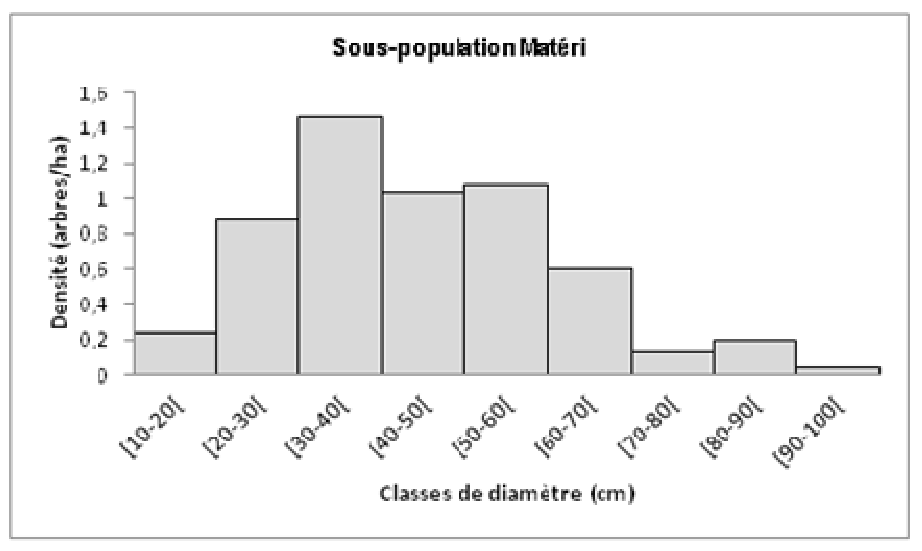

Figure 6 : Structure diamétrique des pieds de $P$. biglobosa de la sous-population Matéri.

\section{DISCUSSION}

Variation morphologique et production fruitière de la population de néré du NordBénin

La présente étude a permis de mettre en évidence l'existence de quatre (4) souspopulations de néré dans la zone d'étude, ainsi qu'une variation morphologique de l'espèce d'une sous-population à une autre. Cette variation morphologique concerne principalement la longueur de la gousse, le nombre de gousses par infrutescence, l'épaisseur du pédoncule des infrutescences, la longueur du pédicelle des gousses, la longueur du pédoncule des infrutescences, le nombre de rachis par feuille, le nombre de graines par gousse et le poids de la gousse. Les présents résultats rejoignent et complètent ceux de Gbédji (2003) qui a observé une variation de la hauteur totale, de la hauteur fût, de la longueur du pédoncule, de la longueur de la gousse, du diamètre à hauteur de poitrine, du diamètre cime et du nombre de graines par gousse, entre les pieds de néré des régions de Bohicon, Savè, Parakou, Bembérèkè et Kandi. Hopkins (1983) a également observé une variation des feuilles et des foliolules de Parkia biglobosa suivant le gradient latitudinal. Au niveau des peuplements de Parkia biglobosa de l'Afrique de l'Ouest et de l'Afrique Centrale (Mali, Sénégal, Niger, Tchad, Burkina-Faso), Ouédraogo (1995) a aussi observé une variation de la hauteur totale, de la hauteur fût et du diamètre cime.

Chacun des descripteurs quantitatifs de la présente étude varie de façon hautement significative d'une sous-population à une autre. Ceci rejoint les résultats d'études antérieures réalisées sur d'autres espèces fruitières telles que Dacryodes edulis (G. Don) H. J. Lam (Leakey et al., 2002a; Waruhiu et al., 2004 ; Anegbeh et al., 2005, Makueti et al., 2012), Irvingia gabonenis (Aubry-Lecompte ex O'Rorke) Baill. ex Lanen (Atangana et al., 2001), Sclerocarya birrea (A. Rich) Hochst. subsp. caffra (Leakey et al., 2002b, Gouwakinnou et al., 2011), Detarium microcarpum Guill. and Perr. (Kouyaté and Van Damme, 2002), Ziziphus mauritiana Lam. (Koné et al., 2009), Allanblackia floribunda Oliver (Atangana, 2010), Adansonia digitata L. (Assogbadjo et al., 2006, 2008, 2009 ; Kyndt et al., 2009 ; Kouyaté et al., 2011), Milicia excelsa (Welw.) C. C. Berg (Ouinsavi et al., 2010), Lagenaria siceraria (Molina) Standl. (Achigan Dako et al., 2008), Vitellaria paradoxa C. F. Gaertn. (Sanou et al., 2006), Canarium indicum L. (Leakey et al., 2008).

En ce concerne l'importance pratique de ce travail, notons avant tout que l'aspect du néré le plus recherché est sa production fruitière. Le présent travail a permis de déceler les sous-populations qui produisent plus de fruits. Les valeurs moyennes de 
nombre de gousses par infrutescence les plus élevées se retrouvent au niveau des souspopulations Kalalé, Sinendé et Matéri. Les individus de néré de la sous-population Kalalé présentent des valeurs moyennes élevées de poids de gousse, nombre de graines par gousse et nombre de gousses par infrutescence. Ils sont donc particulièrement intéressants et peuvent être pris en compte dans le cadre d'une sélection de variétés à haut rendement.

\section{Caractéristiques structurales des sous- populations}

Il ressort aussi de la présente étude que parmi les paramètres dendrométriques étudiés, le diamètre de l'arbre de surface terrière moyenne (Dg), la hauteur totale et la hauteur fût varient significativement d'une souspopulation à une autre. Les individus de néré de la sous-population Kalalé qui produisent plus de fruits présentent des valeurs élevées du diamètre de l'arbre de surface terrière moyenne $(55,95 \mathrm{~cm})$ et de la hauteur totale $(14,55 \mathrm{~m})$. Par contre, leur hauteur fût n'est pas élevée $(2,04 \mathrm{~m})$. Ceci peut être dû au fait que ces arbres de néré se retrouvent dans des milieux complètement ouverts ou dans les champs de mil et d'igname entretenus par les paysans. Souvent ils sont associés à d'autres espèces telles que Vitellaria paradoxa C. F. Gaertn., Anacardium occidentale L., Adansonia digitata L., Mangifera indica L., Borassus aethiopum Mart., etc. Or, ce genre de milieu influence grandement la morphologie du néré qui n'aime pas la compétition. La structure diamétrique des différentes sous-populations de Parkia biglobosa montre une faible densité des pieds de néré dans les classes inférieures de diamètre. Ainsi, en matière de gestion des sous-populations de Parkia biglobosa ces structures diamétriques montrent une insuffisance de la régénération de ces souspopulations. Ouédraogo (1995) avait déjà noté une faible, voire une absence de régénération de Parkia biglobosa dans la partie méridionale de son aire de répartition en Afrique. Selon ce même auteur, cette faible régénération constitue une menace actuelle pour la conservation de l'intégrité du pool génétique de certains allèles rares. Cette faible régénération de Parkia biglobosa est due aux méthodes de prélèvement non appropriées et excessives, aux aléas climatiques et le manque d'alternatives pour les ménages à compenser le déficit des récoltes (Barmo, 2008). Ceci les oblige à exercer une pression de plus en plus forte sur les ressources naturelles. Il est donc important d'envisager l'enrichissement des parcs à néré par plantation de plants élevés en pépinière et la protection de la régénération contre les feux de végétation, le broutage et le piétinement des animaux.

Facteurs explicatifs de la variation morphologique de la population de néré du Nord-Bénin

La présente étude a permis de faire une étude des caractères morphologiques de Parkia biglobosa. En effet, les caractères morphologiques sont très importants et leur étude revêt un intérêt certain (Ouédraogo, 1995). L'analyse des données morphologiques permet d'identifier et de caractériser des groupes de diversité et de préciser leur constitution. C'est ainsi que les souspopulations de néré de la zone étudiée ont été identifiées et caractérisées. L'existence de variations morphologiques au niveau des sous-populations d'une espèce peut s'expliquer par des variations écologiques et génétiques (Kouyaté, 2005 ; Sanou et al., 2006 ; Diallo et al., 2008 ; Assogbadjo et al., 2011 ; Fandohan et al., 2010 ; Gouwakinnou et al., 2011). Une caractérisation des souspopulations de néré identifiées dans la présente étude s'avère donc nécessaire sur le plan climato-pédologique et sur le plan génétique.

Les variations morphologiques constituent apparemment le résultat d'une adaptation à l'environnement. Les facteurs environnementaux (types de sol, texture et caractéristiques chimiques du sol, drainage, précipitations annuelles, compétition liée à la densité de la végétation, etc.) ont eu une grande importance dans la discrimination des 
populations d'espèces végétales notamment les populations de Milicia excelsa (Welw.) C. C. Berg du Bénin (Ouinsavi et al., 2010), les populations de Stenocereus stellatus (Pfeiff.) Riccob. au Centre du Mexique (Casas et al., 1999), les populations de Quercus petraea (Matt.) Liebl. en Italie (Bruschi et al., 2003).

Les variations morphologiques résultent non seulement de facteurs écologiques mais aussi de facteurs génétiques. Des études de diversité génétique et flux génétique de Parkia biglobosa dans les différentes zones agroécologiques du NordBénin sont nécessaires pour expliquer toute la variation phénotypique observée dans la présente étude.

La prise en compte des facteurs environnementaux et génétiques dans la description du niveau et de la structure de la variation phénotypique de la population de Parkia biglobosa du Nord-Bénin permettra de dégager des implications sur la sélection, la conservation et l'amélioration génétique de l'espèce.

\section{Conclusion}

L'étude des caractéristiques phénotypiques et structurales des souspopulations de néré dans le Nord-Bénin nous a permis de dégager quatre sous-populations de néré. Les caractères morphologiques de l'espèce tels que la longueur de la gousse, le nombre de gousses par infrutescence, l'épaisseur du pédoncule, la longueur du pédicelle, la longueur du pédoncule, le nombre de rachis, le nombre de graines et le poids de la gousse varie d'une souspopulation à une autre.

L'étude des structures diamétriques des pieds de Parkia biglobosa des différentes sous-populations montre que la régénération naturelle de l'espèce est faible dans la zone d'étude. Les jeunes individus sont peu représentés. Les jeunes pousses de Parkia biglobosa ne parviennent pas toujours à la maturité car ils sont très vulnérables face aux actions humaines et à d'autres facteurs naturels. Ainsi, en matière de gestion des sous-populations de Parkia biglobosa, les structures diamétriques montrent une insuffisance de la régénération de ces souspopulations. Des dispositions doivent être prises pour assurer la régénération afin de ne pas compromettre, à terme, l'utilisation durable de l'espèce. Aussi :

- Une prise en compte de Parkia biglobosa s'avère nécessaire dans les campagnes annuelles de reboisement qu'organise le gouvernement béninois, afin de résoudre le problème de régénération que rencontre l'espèce. Pour cela, des travaux plus poussés sur la sylviculture de cette espèce doivent être menés.

- Une sensibilisation des paysans est également nécessaire pour une meilleure protection de l'espèce dans les champs, qui offre mieux la possibilité de survie à l'espèce.

- La création des structures de conservation des semences de Parkia biglobosa est aussi indispensable pour assurer sa pérennité.

- Une étude des facteurs écologiques (sol, géomorphologie, climat, etc.) ainsi que des facteurs génétiques qui influencent la variation morphologique de Parkia biglobosa urge dans la zone étudiée.

\section{REMERCIEMENTS}

Nous remercions l'Université d'Abomey-Calavi à travers le projet de fonds compétitifs «Néré 2ADE » pour l'appui financier.

\section{REFERENCES}

Achigan Dako EG, Vodouhè SR, Sangare A. 2008. Caractérisation morphologique des cultivars locaux de Lagenaria siceraria (Cucurbitaceae) collectés au Bénin et au Togo. Belg. J. Bot., 141(1): 21 - 38.

Agbahungba G, Depommier D. 1989. Aspects du parc à karités-nérés (Vitellaria paradoxa Gaertn. F. - Parkia biglobosa Jacq. Benth.) dans le sud du Borgou (Bénin). Bois et Forêts des Tropiques, 222: 41-54.

Anegbeh P, Ukafor V, Usoro C, Tchoundjeu Z, Leakey RRB, Schreckenberg K. 2005. Domestication of Dacryodes edulis: 1. Phenotypic variation of fruit traits from 
100 trees in southeast Nigeria. New Forests, 29(2): 149-160.

Assogbadjo AE, Kyndt T, Sinsin B, Gheysen G, Van Damme P. 2006. Patterns of genetic and morphometric diversity in baobab (Adansonia digitata L.) populations across different climatic zones of Benin (West Africa). Ann. Bot., 97: 819-830.

Assogbadjo AE, Glèlè Kakaï R, Chadare FJ, Thomson L, Kyndt T, Sinsin B, Van Damme P. 2008. Folk classification, perception and preferences of baobab products in West Africa: consequences for species conservation and improvement. Econ. Bot., 62(1): 74-84.

Assogbadjo AE, Kyndt T, Chadare FJ, Sinsin B, Gheysen G, Eyog-Matig O, Van Damme P. 2009. Genetic fingerprinting using AFLP cannot distinguish traditionally classified baobab morphotypes. Agrofor. Syst., 75: 157165.

Assogbadjo AE, Glèlè Kakaï R, Edon S, Kyndt T, Sinsin B. 2011. Natural variation in fruit characteristics seed germination and seedling growth of Adansonia digitata L. in Benin. New Forests, 41: 113-125.

Atangana AR, Tchoundjeu Z, Fondoun J-M, Asaah E, Ndoumbe M, Leakey RRB. 2001. Domestication of Irvingia gabonensis: 1. Phenotypic variation in fruits and kernels in two populations from Cameroon. Agroforestry Systems, 53(1): 55-64.

Atangana AR. 2010. Phenotypic diversity in fruit and seed traits and neutral genetic diversity in Allanblackia floridunda. $\mathrm{PhD}$ dissertation, University of Laval, Quebec, Canada.

Azokpota P, Hounhouigan DJ, Nago MC. 2006. Microbiological and chemical changes during the fermentation of African locust bean (Parkia biglobosa) to produce afitin, iru and sonru, three traditional condiments produced in Benin. International Journal of Food Microbiology, 107: 304-309.
Baco MN. 2007. Gestion locale de la diversité cultivée au Nord Bénin : éléments pour une politique publique de conservation de l'agrobiodiversité de l'igname (Dioscorea spp.). Thèse de doctorat en Socioanthropologie, Université d'Orléans, 406 p.

Barmo S. 2008. Analyse socio - économique de l'exploitation des ressources végétales de la réserve totale de faune de Tamou (Niger). Mémoire de DEA, Biologie appliquée, $88 \mathrm{p}$.

Bonkoungou EG. 1987. Monographie du néré, Parkia biglobosa (jacq.) Benth., espèce agroforestière à usages multiples. Institut de Recherche en Biologie et Ecologie Tropicale. IRBET /CNRST, Ouagadougou, Burkina Faso, 42 p.

Bruschi P, Vendramin GG, Bussotti F, Grossoni P. 2003. Morphological and molecular diversity among Italian populations of Quercus petraea (Fagaceae). Annals of Botany, 91(6): 707-716.

Buisson F. 1965. Plantes Alimentaires de l'Ouest Africain: Etude Botanique, Biologique et Chimique. Leconte: Marseille, France ; 568 p.

Casas A, Caballero J, Valiente-Banuet A, Soriano JA, D'avila P. 1999. Morphological variation and the process of domestication of Stenocereus stellatus (Cactaceae) in Central Mexico. American Journal of Botany, 86(4): 522-533.

Diallo BO, McKey D, Chevalier M-H, Joly HI, Hossaert-McKey M. 2008. Breeding system and pollination biology of the semi-domesticated fruit tree, Tamarindus indica L. (Leguminosae:Ceasalpinideae): implications for fruit production, selective breeding, and conservation of genetic resources. African Journal of Biotechnology, 7(22) : 1068-1075.

Djakpo O. 2005. Fermentation contrôlée des graines de néré (Parkia biglobosa) pour la production d'un condiment béninois de type afitin : effets de l'utilisation des souches sélectionnées de Bacillus subtilis sur la qualité du produit. Thèse 
d'Ingénieur Agronome, Université d'Abomey-Calavi, 72 p.

Duplat P, Perrote G. 1981. Inventaire et estimation de l'accroissement des peuplements forestiers. Office National des Forêts. Section technique. 432 p.

Eyog-Matig O, Adjanohoun E, de Souza S, Sinsin, B. 1999. Réseau «Espèces Ligneuses Médicinales». Compte rendu de la première réunion du Réseau, tenue les 15-17 décembre 1999 à la Station IITA Cotonou, Bénin. Institut International des Ressources Phytogénétiques, $141 \mathrm{p}$.

Eyog-Matig O, Gaoué OG, Dossou B. 2002. Réseau «Espèces Ligneuses Alimentaires ». Compte rendu de la première réunion du Réseau tenue les 11-13 décembre 2000 au CNSF Ouagadougou, Burkina Faso. Institut International des Ressources Phytogénétiques, 235 p. + annexe.

Fandohan B, Assogbadjo AE, Kakaï RG, Kyndt T, Sinsin B. 2010. Quantitative morphological descriptors confirm traditionally classified morphotypes of Tamarindus indica L. fruits. Genetic Resources and Crop Evolution, 58: 299309.

FAO. 2001. Evaluation des ressources en produits forestiers non ligneux.

FAO. 2010. Situation des Forêts du Monde. FAO : Rome, Italie, 110 p. + annexes.

Frankie GW, Baker HG, Opler PA. 1974. Tropical Plant Phenology: Applications for study in community ecology. In Phenology and Seasonality Modeling Lieth H (ed). Springer-Verlag: Berlin; 287-296.

Gbédji EKY. 2003. Caractérisation morphologique et structurale des parcs à nérés (Parkia biglobosa (Jacq.) R. Br. Ex. G. Don.) au Bénin. Thèse d'Ingénieur Agronome, Université d'Abomey-Calavi, $123 \mathrm{p}$.

Gouwakinnou GN, Assogbadjo AE, Lykke AM, Sinsin B. 2011. Phenotypic variations in fruits and potential for selection in Sclerocarya birrea subsp. birrea. Scientia Horticulturae, 129: 777 783.

Gutierrez ML. 2000. Production et commercialisation de l'afitin fon dans la région d'Abomey-Bohicon au Bénin. CERNA, CNEARC, CIRAD : Etudes et Travaux $\mathrm{n}^{\circ} 19,114$ p. + annexes.

Gutierrez ML, Juhé-Beaulaton D. 2002. Histoire du parc à Néré (Parkia biglobosa Jacq. Benth.) sur le plateau d'Abomey (Bénin) : de sa conservation pour la production et la commercialisation d'un condiment, l'afitin. Cahiers d'Outre-Mer, Bordeaux, 220: 453-474.

Hill T, Lewicki P. 2006. Statistics: Methods and Applications, A Comprehensive Reference for Science. Industry and Data Mining, Tulsa: Statsoft, Inc.

Hopkins HC. 1983. The taxonomy, reproductive biology and economic potential of Parkia (Leguminosae : Mimosoidae) in Africa and Madagascar. Bot. J. Linn. Soc., 87: 135-167.

Hounhouigan DJ, Ahouansou R, Madode Y. 2004. Test d'adaptation en station d'une décortiqueuse de néré pour la production de «Afitin» au Bénin. Rapport de recherche PADSA, $10 \mathrm{p}$.

Kyndt T, Assogbadjo AE, Hardy OJ, Glele Kakaı. R, Sinsin B, Van Damme P, Gheysen G. 2009. Spatial and temporal genetic structuring of Adansonia digitata L. (Malvaceae) in the traditional agroforestry systems of West Africa. Am. J. Bot., 96(5): 950-957.

Koné B, Antoine Kalinganire A, Doumbia M. 2009. La culture du jujubier : un manuel pour l'horticulteur sahélien. ICRAF Technical Manual no. 10,, World Agroforestry Centre : Nairobi.

Koura K. 2003. Contribution à l'étude ethnobotanique du néré [Parkia biglobosa (Jacq.) R. Br. ex G. Don] dans les départements de l'Atacora et de la Donga : Aspects socioculturels. Mémoire de DESS en Aménagement et Gestion des Ressources Naturelles option Sciences et Techniques Forestières, FSA / UAC, 94 p. + annexes. 
Kouyaté AM, Van Damme P. 2002. Caractères morphologiques de Detarium microcarpum Guill. et Perr. au sud du Mali. Fruits, 57: 231-238.

Kouyaté AM. 2005. Aspects ethnobotaniques et étude de la variabilité morphologique, biochimique et phénologique de Detarium macrocarpum Guill. \& Perr. au Mali. Thèse présentée pour l'obtention du grade de Doctorat $(\mathrm{PhD})$ en Biosciences Ingénieurs, section Agronomie. Université de Ghent, Belgique, p. 207.

Kouyaté AM, Decaluwé E, Guindo F, Diawara H, Diarra I, N'Diayé I, Van Damme P. 2011. Variabilité morphologique du baobab (Adansonia digitata L.) au Mali. Fruits, 66: 247-255.

Lafleur M. 2008. Recherches et Documentation des Meilleures Pratiques pour la Gestion Durable des Parcs en Karité en Afrique de l'Ouest. CECI : Montréal, 110 p.

Leakey RRB, Atangana AR, Kengni E, Waruhiu AN, Usoro C, Tchoundjeu Z, Anegbeh PO. 2002a. Domestication of Dacryodes edulis in West and Central Africa: characterization of genetic variation. Forests Trees and Livelihoods, 12: $57-71$.

Leakey RRB, Shackleton S, Du Plessis P, Pate K, Lombard C. 2002b. Characterization of phenotypic variation in marula (Sclerocarya birrea) fruits, nuts and kernels in South Africa and Namibia. DFID/FRP: Winners and Losers in Forest Product Commercialization, Project $\mathrm{N}^{\circ}$ ZF0140/R7795.

Leakey RRB, Fuller S, Treloar T, Stevenson L, Hunter D, Nevenimo T, Binifa J, Moxon J. 2008. Characterization of treeto-tree variation in morphological, nutritional and medicinal properties of Canarium indicum nuts. Agroforestry Systems, 73: 77-87.

Makueti JT, Tchoundjeu Z, Kalinganire A., Nkongmeneck BA, Kouodiekong L, Asaah E, Tsobeng A. 2012.
Morphological traits of control-pollinated fruits in African plum (Dacryodes edulis (G. Don) H. J. Lam.) using multivariate statistical techniques. IJAAR, 2(8): 1-17.

Ouédraogo A-S. 1995. Parkia biglobosa (Leguminosae) en Afrique de l'Ouest: Biosystématique et Amélioration. Thèse de doctorat de l'Université Agronomique de Wageningen, Institute for Forestry and Nature Research, IBN-DLO, Wageningen, Netherlands, 205 p.

Ouinsavi C, Sokpon N. 2010. Morphological Variation and Ecological Structure of Iroko (Milicia excelsa Welw. C.C. Berg) Populations across Different Biogeographical Zones in Benin. International Journal of Forestry Research, 10 p, DOI:10.1155/2010/ 658396.

Rateau N. 1995. Etude de la valeur nutritionnelle du néré ou « Parkia biglobosa ». Mémoire de DESS en nutrition et alimentation dans les pays en développement, Université des Sciences, Montpellier II, France.

Schmidt-Soltau K, Alimi RM. 2008. Programme National de Gestion Durable des Ressources Naturelles. Rapport Final. République du Bénin. 55 p. + annexes.

Sina S. 2006. Reproduction et diversité génétique chez Parkia biglobosa (Jacq.) G. Don. $\mathrm{PhD}$ thesis Wageningen University, $102 \mathrm{p}$.

Sanou H, Picard N, Lovett PN, Dembélé M, Korbo A, Diarisso D, Bouvet J-M. 2006. Phenotypic variation of agromorphological traits of the she butter tree (Vitellaria paradoxa C.F. Gaertn.) in Mali. Genetic Resources and Crop Evolution, 53: 145-164.

Waruhiu AN, Kengue J, Atangana AR, Tchoundjeu Z, Leakey RRB. 2004. Domestication of Dacryodes edulis 2: Phenotypic variation of fruits in 200 trees from four populations in the humid lowlands of Cameroon. Food Agriculture and Environment, 2(1): 340-346. 